[2] Kuo C-F, Grainge MJ, Zhang W, Doherty M. Global epidemiology of gout: prevalence, incidence and risk factors. Nature Reviews Rheumatology 2015;11:649.

[3] Mehta T, Nuccio E, McFann K, Madero M, Sarnak MJ, Jalal D. Association of Uric Acid With Vascular Stiffness in the Framingham Heart Study. Am J Hypertens 2015;28:877-83.

Disclosure of Interests: None declared

DOI: 10.1136/annrheumdis-2019-eular.4444

\section{SAT0427 RISK OF MALIGNANCIES IN PATIENTS WITH GOUT: A POPULATION-BASED COHORT STUDY}

Oh Chan Kwon, Hyun Ah Lee, Seokchan Hong, Chang-Keun Lee, Bin Yoo, Ji Seon Oh, Yong-Gil Kim. University of Ulsan College of Medicine, Asan Medical Center, Seoul, Korea, Rep. of (South Korea)

Background: Gout is a chronic disease characterized by deposition of monosodium urate crystals. Comorbidities including hypertension, chronic kidney disease, obesity, diabetes and cardiovascular diseases are common in patients with gout. In contrast to these well-known comorbidities, little is known about the risk of malignancies in patients with gout.

Objectives: To investigate the risk of malignancies in patients with gout, compared with general population.

Methods: We conducted a retrospective cohort study using Korean National Health Insurance Service-Medical check-up Cohort Database, which composed of qualified individuals as of 2002 in the age of 40-79 in 2002-2003 who received general medical check-up (Approximately $510,000)$. We enrolled patients newly diagnosed with gout, based on the diagnostic code and relevant medication history, who were between 40 and 65 years of age at the time of diagnosis between 2003 and 2007 (we washed out first year for newly detected cases). The gout patients (case group) were matched by 1:2 propensity score matching using confounding variables (age, sex, income group, region of residence, smoking status, alcohol intake, exercise habit, comorbidities including diabetes mellitus, hypertension and dyslipidemia, body mass index, blood pressure, serum glucose level, total cholesterol, and hemoglobin) and survival analysis was performed to estimate the risk of malignancy.

Results: A total of 4991 cases and 419992 controls were identified. The prevalence of Gout was 4991 (1.17\%, male 4093 (82.01\%); female 898 $(17.99 \%))$. During a mean follow-up of 12 years, malignancy was newly diagnosed in 30262 patients $(7.12 \%$ of the total cohort). Gout was associated with increased risk of malignancy in the multivariable Cox proportional hazard regression analysis before propensity score matching (hazard ratio (HR) 1.248, 95\% confidence interval $(\mathrm{Cl})$ 1.130-1.379, $\mathrm{p}<0.001)$, as well as after matching (HR 1.369, 95\% Cl 1.209-1.549, $\mathrm{p}<0.001)$.

Conclusion: A total of 4991 cases and 419992 controls were identified. The prevalence of Gout was 4991 (1.17\%, male 4093 (82.01\%); female $898(17.99 \%))$. During a mean follow-up of 12 years, malignancy was newly diagnosed in 30262 patients $(7.12 \%$ of the total cohort). Gout was associated with increased risk of malignancy in the multivariable Cox proportional hazard regression analysis before propensity score matching (hazard ratio $(\mathrm{HR})$ 1.248, 95\% confidence interval $(\mathrm{Cl})$ 1.130-1.379, $\mathrm{p}<0.001)$, as well as after matching ( $\mathrm{HR}$ 1.369, 95\% Cl 1.209-1.549, $\mathrm{p}<0.001)$.

\section{REFERENCES}

Not applicable

Table. Estimation of risk of malignancy using Cox proportional hazard regression analysis

\begin{tabular}{|c|c|c|c|c|c|c|}
\hline & \multicolumn{3}{|c|}{ Before propensity score matching Cox } & \multicolumn{3}{|c|}{ After propensity score matching Cox } \\
\hline & HR & $95 \% \mathrm{Cl}$ & $P$ & $\mathrm{HR}$ & $95 \% \mathrm{Cl}$ & $P$ \\
\hline Gout & 1.248 & $1.130-1.379$ & $<0.001$ & 1.369 & $1.209-1.549$ & $<0.001$ \\
\hline
\end{tabular}

Disclosure of Interests: None declared

DOI: 10.1136/annrheumdis-2019-eular.6583

\section{SAT0428 \\ ANALYSIS OF THE PREVALENCE AND TIMING OF GOUT CO-MORBIDITY IN PATIENTS UNDERGOING KIDNEY TRANSPLANT}

Brian LaMoreaux, Megan Francis-Sedlak, Robert J. Holt. Horizon Pharma USA, Inc., Lake Forest, United States of America

Background: Patients receiving kidney transplants are at increased risk for the development of hyperuricemia and gout compared to the general population, which is generally attributed to the frequent use of calcineurin inhibitors (cyclosporine and tacrolimus). However, the precise proportion of renal transplant patients that develop gout and the time period post-transplant in which this occurs is less established.

Objectives: To analyze a large, mixed-insurance, US population database to determine gout prevalence in the renal transplant population.

Methods: A retrospective review of Humana Healthcare claims data (private and Medicare) from 2007 to 2017 was undertaken to identify kidney transplant patients with at least 6 months in plan prior to transplant and at least 6 months in plan post-transplant. Diagnosis of gout $(\geq 1$ gout ICD $10 /$ ICD 9 code) was evaluated in relationship to the time of kidney transplant.

Results: The database contained 6,082 patients with a kidney transplant and at least 6 months in plan both pre and post-transplant. Of the 6,082 kidney transplant patients, $1,505(25 \%)$ had a gout diagnosis: 908 (15\% of transplant patients) with gout before and after transplant and 597 (10\% of transplant patients) with a gout code only after transplant. In patients developing gout post-transplant, the mean time between transplant and gout diagnosis was $1.79 \pm 1.85$ years.

Conclusion: As expected, gout was a common comorbidity in renal transplant patients. $15 \%$ of the patients receiving renal transplants had gout prior to the transplant, and another $10 \%$ developed new-onset gout a mean of 1.79 years after receiving a renal transplant. This retrospective analysis demonstrates that kidney transplant patients commonly suffer from gout both before and after their transplant. In addition to more research on this topic, an increased focus on screening and treatment of gout in the renal transplant population may be warranted.

\section{REFERENCES}

[1] Abbott K.C., Kimmel P.L., Dharnidharka V., et al. Transplantation. 2005 80:1383-1391

[2] Zhu Y., Pandya B.J., Choi H.K. Arthritis \& Rheumatism. 2011; 63 (10):3136-3141

Disclosure of Interests: Brian LaMoreaux Shareholder of: Horizon Pharma, Employee of: Horizon Pharma, Megan Francis-Sedlak Shareholder of: Horizon Pharma, Employee of: Horizon Pharma, Robert J Holt Shareholder of: Horizon Pharma, Employee of: Horizon Pharma

DOI: 10.1136/annrheumdis-2019-eular.2893

\section{SAT0429 COMORBIDITIES IN AN EARLY DIAGNOSED COHORT OF UNCONTROLLED VERSUS CONTROLLED GOUT: ANALYSIS OF A LARGE US PAYER DATABASE}

Megan Francis-Sedlak, Brian LaMoreaux, Robert J. Holt. Horizon Pharma USA, Inc., Lake Forest, United States of America

Background: Gout is a widely prevalent progressive systemic inflammatory arthritis. The pathogenic cause of gout is elevated serum uric acid or hyperuricemia, and appropriate treatment of gout involves reduction of uric acid levels to a minimum goal of less than $6 \mathrm{mg} / \mathrm{dL}$. Patients who do not achieve uric acid goals are generally described as uncontrolled gout patients and tend to do worse in terms of clinical outcomes such as occurrence of flares and persistence/worsening of tophi. Gout patients often suffer from specific comorbidities, though whether uncontrolled gout patients have a different comorbidity profile is unclear.

Objectives: The objectives of this evaluation were to compare the comorbidities and hospitalizations in uncontrolled versus controlled gout patients from a large de-identified US payer database.

Methods: A retrospective review of Humana Healthcare data from 2007 to 2016 in private pay and Medicare patients was performed to identify 